OPEN ACCESS

Edited by:

Christothea Herodotou, The Open University, United Kingdom

Reviewed by: Michele Meoli, University of Bergamo, Italy Peter Jones, University of Gloucestershire, United Kingdom

${ }^{*}$ Correspondence: Eva Y. W. Wong evawong@associate.hkbu.edu.hk

Specialty section:

This article was submitted to Digital Learning Innovations, a section of the journal Frontiers in Education

Received: 15 October 2020

Accepted: 22 July 2021 Published: 17 September 2021

Citation: Leung FYW, Lau M, Wan K, Law L, Kwong $T$ and Wong EYW (2021)

Promoting Students' Global Perspectives Through a Gamified e-

Learning Platform.

Front. Educ. 6:617680.

doi: 10.3389/feduc.2021.617680

\section{Promoting Students' Global Perspectives Through a Gamified e-Learning Platform}

Frankie Y. W. Leung, Martin Lau, Kelvin Wan, Lisa Law, Theresa Kwong and Eva Y. W. Wong *

Centre for Holistic Teaching and Learning, Hong Kong Baptist University, Hong Kong, China

With the rapid growth of internationalization in tertiary institutions worldwide, the development of students' global perspectives has attracted the attention of many universities. However, this development is a challenging one due to the complicated nature of global issues and their incompatibility with traditional subject-specific boundaries of classroom teaching. Through two eTournaments organized on a proprietary gamified e-learning platform named "PaGamO," this study examined participating students' learning experience and their change of global perspectives due to their participation in the eTournaments. Data were collected before and after the two eTournaments, and 217 survey responses were considered to be valid and were further analyzed. The findings showed that participating students achieved the satisfaction level of enjoyment $(M=3.62)$ and their awareness of the United Nations Sustainable Development Goals (SDGs) $(M=$ 3.96) had been improved. In addition, the findings also revealed that 1) students enjoyed and perceived a better understanding of the SDGs in terms of perceptual dimensions like value-oriented and partnership-oriented, rather than the global issues about substantial threats or environmental issues; 2) the "intrapersonal effect" of students had been significantly reduced after the eTournaments; 3) positive significant correlations were found between the level of enjoyment and frequency of question-attempt in relation to the change of cognitive knowledge and interpersonal social interaction. The findings of this study offered some possible insights into students' gameplay experience concerning dimensions of global perspectives and also support the findings of prior research on how gamified e-learning platforms could contribute to the development of students' global perspectives.

Keywords: gamified learning, global perspective, online learning, e-learning, sustainable development goals

\section{INTRODUCTION}

Internationalization in higher education has been accelerating rapidly in the past 40 years (Guo et al., 2021). This "process" (Knight, 2004) increasingly impacts higher education institutions in the world, which facilitates research collaborations, staff and students' mobility, and cultural and economic exchanges (Seeber et al., 2020). While internationalization could be applied to a multi-level organization and sectors, internationalization in higher education could be generally defined as "the process of integrating an international, intercultural or global dimension into the purpose, functions or delivery of post-secondary education" (Knight, 2004). The definition of internationalization had been modified several times (Knight, 1994, Knight, 2004; Guo et al., 
2021), and the recent outbreak of COVID-19 has led scholars to believe that the current framework is incompatible (Taşçı, 2021). However, these revised definitions and approaches (e.g., competency approach) remained linked and "complementary" (Knight, 2004), reflecting on the dynamic shifts in the "complex world" (Leask, 2015, 27). The reform of definitions may continue as time passes (de Wit and Altbach, 2021), but the core rationale of internationalization emphasizes a sense of relationships between nations, diverse cultures, and global issues at all levels, including institutional, local, national contexts.

From an individual perspective, one of the approaches in describing internationalization is the competency approach, which emphasizes how to develop internationalization in terms of knowledge, skills, and attitudes among students in the globalized world (Knight, 1994). Graduate attributes are a typical example of this approach to link up with the concepts of internationalization and bringing theories into the practices, as the internationalization of curriculum (Leask, 2015, 53). While this approach addresses human growth rather than organizational changes, many institutions use the term "global perspectives" and "international perspectives" explicitly in their websites to represent this as a generic skill for recognizing and respecting the inter-connection of life in a globalized world (Leask, 2015, 55).

Internationalization is frequently viewed from a global perspective rather than as a regional issue (Braskamp, 2009). It is because through understanding the interconnectedness of global issues, students could develop more capacities to address such problems and increase their own competitiveness in the economy (Baildon et al., 2018). It is therefore vital for university education to prepare students to become future leaders with global perspectives, which enable them to address the world's pressing challenges and improve their intercultural social skills.

Although the importance of possessing internationalization through global perspectives had been highlighted by previous works (Warner, 2017; Medora et al., 2020), the term "global perspective" is often considered as an alternative learning outcome of a program rather than an independent learning task (Smith and Yang, 2017) to be achieved by students. Furthermore, most studies conducted about the promotion of global perspectives mainly focused on the teacher-led internationalization activities, such as Education Abroad Program (Hudson and Tomás-Morgan, 2019), video conferencing (Greenwood, Honey, and Clancy, 2016), or university culture (Shephard, Bourk, Mirosa, and Dulgar, 2016), and did not address how students acquired their global perspectives through self-directed learning.

Internationalization can be accomplished through emerging knowledge from cross-cultural contexts into the curriculum (Knight, 1994; Seeber et al., 2020). However, integrating global perspectives as an effective means of internationalization into the formal curriculum had faced some challenging issues. First, global perspectives are often undervalued or incompatible with the traditional subject-specific boundaries of classroom teaching. More boundless learning opportunities should be offered to students to widen their horizons, whereas it may affect the professional recognition of the corresponding degree program
(Enonbun, 2010). Second, the selection of global issues is a difficult task-the content should involve the mutual concerns of both developing and developed countries, and an option should be provided to work in a field that crosses all national boundaries, cultures, demographics, and regions (Evans, Ingram, MacDonald, and Weber, 2009). For example, Kopnina (2015) suggested that well-developed countries are more interested in keeping economic growth countries, while their citizenconsumers do not want to scarify themselves for environmental protection, climate change, or other relevant SDGs. Similarly, it is not realistic to ask students from developing countries to talk about "decent work" when they have to face the challenges of survival, like poverty and hunger, every day. It is challenging to integrate the thoughts of students with multidisciplinary and multicultural backgrounds. As a result, despite the importance of possessing global perspectives is highlighted by many researchers, few universities actually dedicate the effort and resources to promote global perspectives on a large scale. Most students could only develop global perspectives themselves through overseas exchange or intercultural interaction at an individual level, without any systematic school coordination (Evans et al., 2009).

Addressing the above concerns, the idea of Internationalization at Home $(\mathrm{IaH})$ was proposed by scholars, which aims to educate students in local learning environments by incorporating international and intercultural components into the formal and informal curricula. This approach, as classified in prior work (Barbosa et al., 2020), is thought to improve "virtual mobility" by providing a technology-assisted environment, an alternate approach for students to collaborate globally, and therefore better promote students' global perspectives. Therefore, this study aims to explore the role of technologyassisted challenge-based learning in promoting students' global perspectives. In this study, a gamified e-learning platform, "PaGamO" was used. It allowed students with multidisciplinary and multicultural backgrounds to work together in teams and compete on a virtual map with their knowledge of the 17 United Nations (UN) Sustainable Development Goals (SDGs). The 17 SDGs were agreed upon by world leaders in 2015 to represent the universal challenges of humans from a global perspective (United Nations, 2015).

\section{LITERATURE REVIEW}

\section{Definition and Dimensions of the Global Perspective}

Global perspective refers to the nature of a study that is globally oriented, instead of the view that is being confined to the cultural or political preferences and inclinations of the particular country (Hua, 2008). Previous researchers tried to give a detailed description of what global perspectives should cover. Some researchers focused on its significant dimension, emphasizing the range of global topics about which people should be informed, such as universal human values that transcend group identity (e.g., equality, justice, and liberty), and persistent global issues 


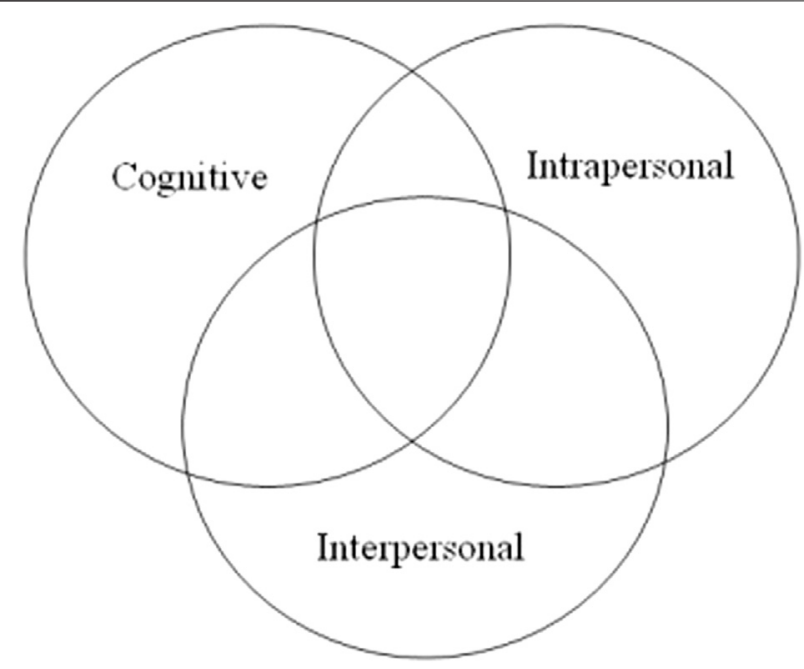

FIGURE 1 | Three dimensions of global perspective.

and problems (e.g., poverty or femininity) (Kniep, 1986). These researchers focused more on knowledge and information, while the development of global perspectives is considered as a cognition process of gaining understanding about different global knowledge, value, and information.

Case (1993) also agreed with the substantial dimension, yet he stepped forward and extended the discussion of global perspectives to "perceptual dimension," which refers to "various intellectual values, dispositions, and attitudes that distinguish a parochial perspective ... from a broad-minded perspective" (P.320). Case (1993) outlined five main perceptions of developing students' global perspectives, namely, 1) open-mindedness; 2) anticipation of complexity; 3) resistance to stereotyping; 4) inclination to empathize; 5) nonethnocentrism (thinking one's group is superior to others). Case's idea is further developed into the concept of intercultural competence, which refers to the "modes of thought, sensitivities, intellectual skills, and explanatory capacities" (Deardorff, 2009, p.443). In this view, the development of global perspectives is not just cognitive but also involves the psychological and emotional development of dispositions, ethical position, open-mindedness, and multicultural attitude. It thus involves how individuals deal with cultural diversity and how to make sense of the world.

Integrating the substantial and perceptual dimensions of the global perspectives, Braskamp et al., 2014 conceptualized them into three domains: cognitive, intrapersonal, and interpersonal (Figure 1).

In Braskamp's view, the cognitive domain is about intercultural knowledge, which includes "individual knowledge and knowing with greater complexity and taking into account multiple cultural perspectives" (Braskamp, 2014, p.3). The intrapersonal domain focuses on individual awareness and how they integrate their own personal values and self-identity internally. The interpersonal domain is more about individual attitude and behavior. It is centered on one's willingness to interact with outgroup cultures and their acceptance of others (Braskamp et al., 2014).

\section{Global Learning for Global Perspectives and SDGs}

At the university level, students' global perspectives could be developed through global learning. Global learning is the learning process where students with diverse cultural backgrounds make collaborative efforts to tackle and resolve the complicated problems that transcend the national borders (Landorf and Doscher, 2015). And it was recognized as one of the ways to aid institutional internationalization $(\mathrm{Ng}$ and Nyland, 2016). Students could develop individual competence through global learning by exploring and considering different points of view.

During the global learning activity, the students would share their own viewpoints as a member of a virtual team, which is known as social presence (Wang, 2009). Also, global learning should help develop global awareness, knowledge of the world's complexity, and interrelatedness. It could also facilitate the students' collaboration since they would acknowledge that they are too complex for any single person, group, discipline, or approach to solve alone.

Although the concept of global perspectives is an individual capacity, its development is not context-independent. There is a need to choose global-awareness issues that the students could explore and integrate different perspectives. In this regard, the 17 SDGs serve as good examples for students to explore their implications.

The 17 SDGs are the core of "The 2030 Agenda for Sustainable Development" of the UN, which serves as a "blueprint to achieve a better and more sustainable future for all," instead of the narrow consideration of the particular country. All SDGs require the collaborative actions of all countries-developed and developing-in a global partnership (United Nations, 2015), such as ending poverty, reducing inequality, or tackling climate change. These SDGs had been well noted in previous literature that could be used as an agent to call upon to help contribute to the challenges and goals in internationalization (de Wit and Altbach, 2021).

Through a prolonged discussion in the UN, the 17 SDGs were agreed upon by all world leaders and were adopted at the UN Sustainable Development Summit in September 2015 (United Nations, 2015). The goals are included in Table 1.

It is worth noting that learning SDGs in the university setting is a complicated task. It often involves a deep conflict when attaining different SDGs at the same time. For example, Hickel (2019) suggested that continued global industrial growth in SDG 8 may not be reconcilable with ecological sustainability goals (SDG 12-15) due to the global eco-economic decoupling in recent years. Also, economic growth (SDG 8) may worsen the goal of inequality reduction or other sustainability objectives. Thus, the implementation of the SDGs needs to balance conflicting positions and compromise, such as exploring the possibility of ecotourism or global recycling industry, which are generally considered as the typical example of seeking a 
balance between economic benefits for resident hosts or consumers' benefits and environmental protection against the additional costs (Alexander and Whitehouse, 2004).

Moreover, the teaching and learning of global perspectives and SDGs is not an easy task. Previous literature showed that most teachers focused on the development of substantial dimensions only. Merryfield, 1998, for example, suggested that most teachers focused on the particular topics of culture and history, such as human rights, the slave trade, or child labor. On the other hand, Kirkwood, 2002 suggested that most teachings about world-mindedness tended to highlight the cultural universals, such as loving families, self-esteem, and personal and cross-cultural appreciation. At the university level, however, the knowledge of people and places is clearly not enough. Although the introductory courses about global perspectives could be the starting point for raising students' awareness of others' perspectives, the lecturer is difficult to help students develop desirable attitudes and sensibilities, such as open-mindedness, tolerance, empathy, or consciousness of their own national orientations, as well as their worldview.

\section{Promoting Global Perspectives Through a Gamified e-Learning Platform}

As mentioned before, one of the challenges of promoting global perspectives is that most global issues are extremely complicated because of their cross-disciplinary, cross-cultural, demographical, and regional nature. Moreover, it does not entirely fit the formal curriculum. Teachers could only use the example of global issues for instruction or to encourage students to apply their professional knowledge to tackle global problems. At the same time, students would consider whether the learning content could benefit their academic achievement or professional recognition. Thus, it is difficult to motivate them and build up their awareness of global perspectives outside the official curriculum.

Addressing the above limitations, a gamified e-learning platform was offered as an informal internationalization curriculum for students. The benefits of gamified ICTenhanced teaching have been frequently reported by different researchers, such as increasing students' motivation and engagement (Koivisto and Hamari, 2019), empowering students with low self-efficacy, and even facilitating the development of critical thinking (Turkay et al., 2014; da Rocha Seixas et al., 2016). Similarly, it is also reported as an effective tool to introduce global perspectives to students beyond the traditional classroom. Previous studies suggested that gamified e-learning platforms are effective in teaching nonsubject knowledge, such as leisure reading (Mak et al., 2019), traffic safety (Riaz et al., 2019), or civic engagement (Hassan, 2017). Not only could the gamified e-learning platform motivate students through the peer-pressure or team collaboration, but it also offers external motivation (i.e., points, achievements, or ranking) that makes students more committed to learning and achieve higher levels of flow experience during the gameplay (Mak et al., 2019).

\section{METHODOLOGY}

As noted above, the need for global perspectives as one of the research indicators of internationalization had been documented earlier (McCabe, 1994; Leask, 2015), and that the intervention was frequently through formal teacher-led activities to promote students' global perspectives. This research bridges the void by using gamified SDGs content to help students develop global perspectives. As a result, this research investigates the effectiveness of using an eTournament on the gamified e-learning platform "PaGamO" to help students gain global perspectives. It also examined students' perceptions of their gameplay experience. A central question of this study is

To what extent could the gamified e-learning platform help students develop their global perspectives?

To guide the study, the following three research questions are proposed:

RQ1. What are students' perceptions of their gameplay experience and improvement of SDG awareness?

RQ2. How do the students' global perspectives change after the eTournament?

RQ3. What is the correlation between students' gameplay experience and the change of global perspectives in the eTournament?

\section{Research Design}

This study adopted a pre-test, post-test quasi-experimental design. A quantitative approach aims to help researchers grasp the pattern of response among the large population (Kendall, 2008). Since this study involved more than two hundred students, the quantitative approach allows us to explore how the gamified e-learning platform works well in the development of students' global perspectives. Second, it allows the researchers to investigate students' feedback from a larger sample size for more generalizable results (Oppenheim, 1992).

\section{The SDGs eTournament}

This study involved two eTournaments; they both had a similar design and both aimed to 1) allow students from different parts of the world to learn to work together online (Online Teams) and complete specific tasks, 2) let students learn about the $17 \mathrm{UN}$ Sustainable Development Goals (SDGs) through the game platform, and 3) allow students to learn about the different cultures and background of their teammates (Hong Kong Baptist University, 2020). Two eTournaments were held in the spring of 2019 and spring of 2020, respectively. Both eTournaments had two stages which will be described in the following.

\section{The Gamified Platform}

"PaGamO" was used to bridge the gap between informal and formal global perspective learning. PaGamO was developed by a professor from the National Taiwan University, which allows players to learn and compete with each other in an online virtual map by answering preset questions. PaGamO allows teachers to 


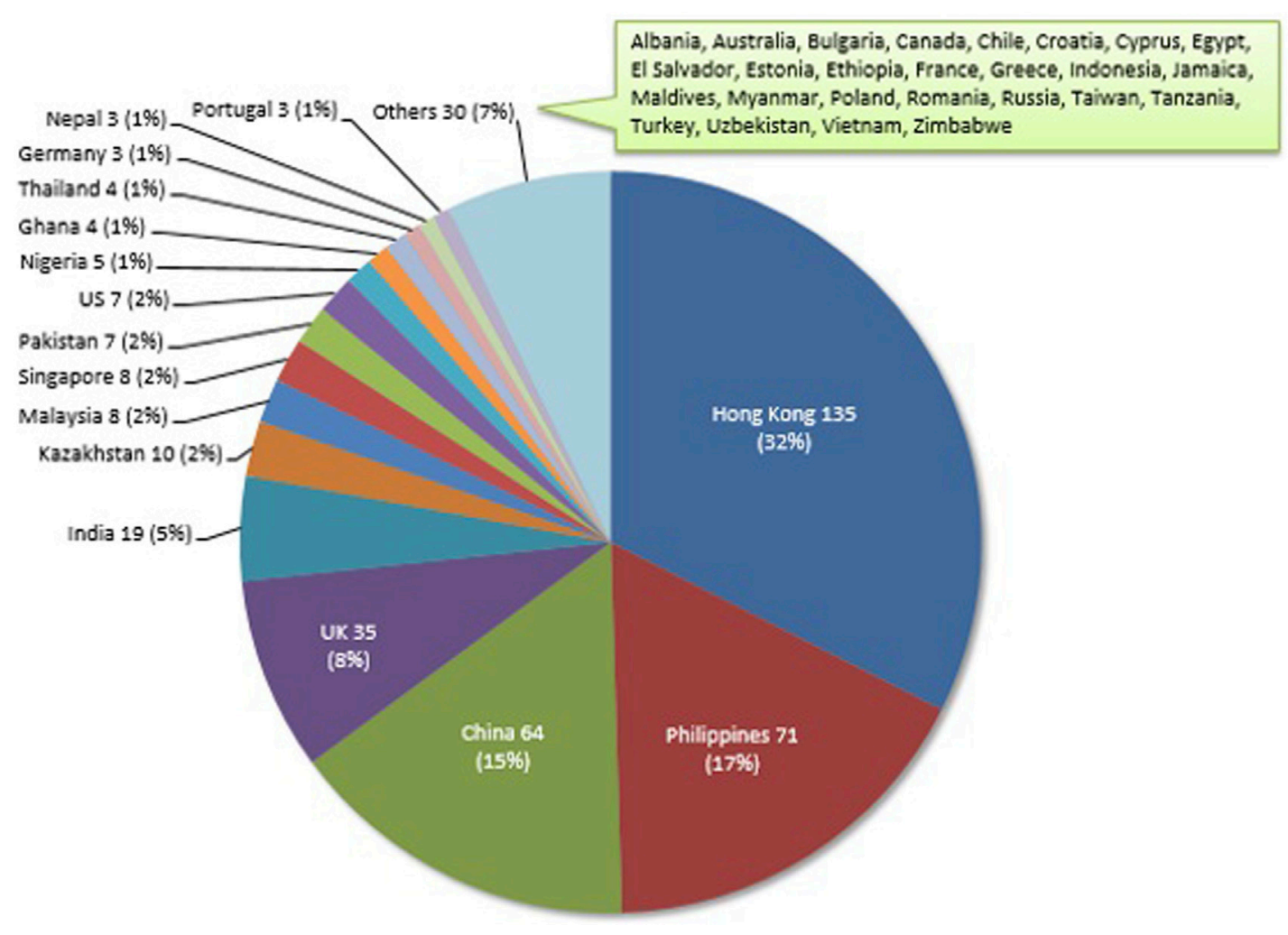

FIGURE 2 | Home region of student participation (second eTournament) $(N=416)$.

prepare their own question bank according to the learning objective (Hong Kong Baptist University, 2020). Also, the PaGamO system provided a convenient way to explore different learning analytic features like the frequency of question attempts for further analysis.

\section{Game Content}

The questions about the SDGs are multiple-choice questions (MCQ) which were developed through two approaches. On the one hand, university students from all over the world were invited to submit SDG questions in the 2018 SDG Questions Creation Contest. The expert judges at UNESCO Hong Kong Association reviewed all questions in the contest to ensure their validity of fitting the learning objective of the global perspectives. In the contest, 706 valid SDGrelated questions were prepared by 117 university students from 10 institutions in Hong Kong, Australia, India, the Philippines, and Singapore. On the other hand, experts from UNESCO Hong Kong Association were invited to develop the SDG questions so that an SDG questions bank of about 1,400 questions was created.

\section{Preparation Stage}

Stage 1 is the preparation stage, where all students were invited to do online discussions in a pre-assigned virtual team so that they could get familiar with each other and work out the strategies for the gameplay. During Stage 1, each team was asked to choose their team leader, PaGamO game characters, and discuss their game strategy for Stage 2.

\section{Game Play Stage}

Stage 2 is the gameplay stage, where teams had to compete with each other on the virtual map of PaGamO by answering SDGs questions. The questions were put into a single question pool and were assigned to students randomly in the first eTournament, while in the second eTournament, the questions of each SDG were put into different sets, and the students could choose to answer particular sets of SDG questions.

\section{Sampling}

With the involvement of four local partner institutions and 13 international collaborators, this study invited students from universities all over the world. In general, all university students, ranging from sub-degree to doctoral level, are eligible to join the eTournament. Each team consisted of four to five members, and all team members were assigned to each group randomly. To ensure student diversity, no more than two students from the same institution or region would be grouped together in each team.

To minimize the non-response bias among students, two criteria were used in the data filtering process. First, the students would not be counted if their frequency of question attempts (the sum of attack-action and land-training action in PaGamO) was less than ten. Second, the students would be considered as having survey fatigue if they made "straight-line responses" (i.e., giving answers down the same column) over fifteen questions. Previous literature suggested that survey fatigue 


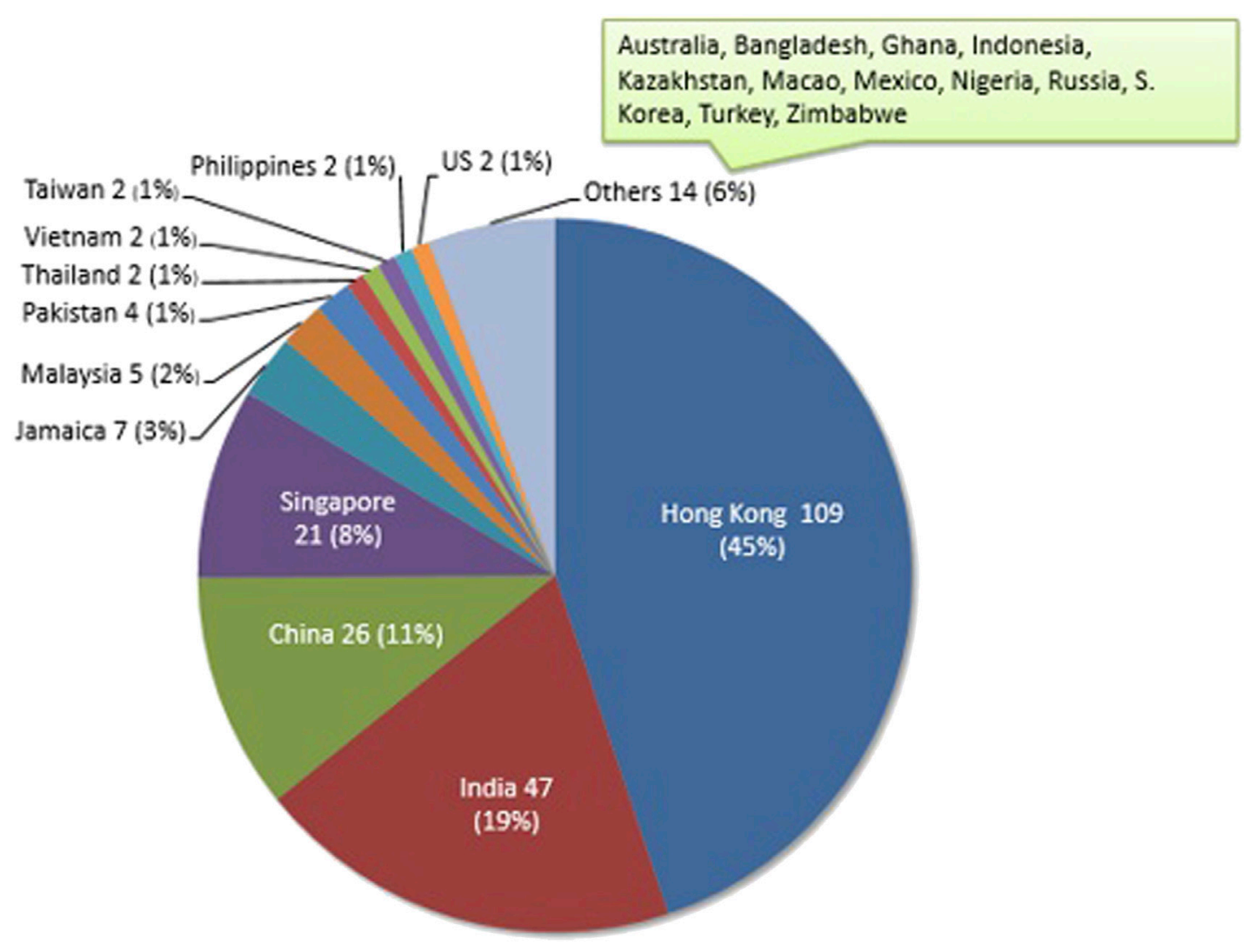

FIGURE 3 | Home region of student participation (first eTournament) ( $N=243$ ).

would lead to non-response bias and cover the significant findings (Lavrakas, 2008).

\section{Data Collection and Instrument}

This study has been approved by the university's Research Ethics Committee. At the first survey, participants volunteered for the study and received their informed consent. Data were collected before and after the eTournament, with 8-day (2019 run) and 16-day (2020 run) gaps between the two surveys. All participating students were invited to fill in the pre-survey while those who finished the final stage of the eTournament were invited to complete the post-survey about their learning experience of global perspectives, level of enjoyment, and perceived improvement of SDG awareness. They would earn some bonus points for the eTournament after completing the survey. Qualtrics, an online survey tool, was used to collect the data. After the eTournament, the question attempts were extracted from $\mathrm{PaGamO}$ as part of the learning analytic function.

The global perspectives were measured by a shortened version of the Global Perspective Inventory (GPI), which was developed by Braskamp and his colleagues in 2014. Since the two eTournaments were brief and had no direct effect on individual behavior or life-long social responsibility, the dimension "Social Responsibility" was excluded from the survey.

The shortened version of GPI in this study consists of 22 items, which categorized global perspectives into five dimensions: 1) cognitive knowing, 2) cognitive knowledge, 3) intrapersonal identity, 4) interpersonal affect, and 5) interpersonal social interaction (Braskamp et al., 2014). A 5-point Likert scale was used in the surveys, and students were invited to comment on each statement according to their experience, with one indicating "strongly disagree" and five indicating "strongly agree."

In the survey, the demographic and other self-reported data such as gender, contact email, level of enjoyment, and perceived improvement of SDG awareness were also collected. A descriptive statistical analysis was performed using the data from PaGamO's question attempts.

\section{Data Analysis}

First, the Cronbach's alpha of each dimension of GPI was calculated in order to ensure internal consistency. Second, addressing RQ1, the descriptive statistical analysis was conducted on the mean scores of 1) level of enjoyment; 2) perceived improvement of SDG awareness; (3) frequency of question-attempt (including the student's attempt to attack others, expand territory, and train his own land), which explicitly indicated students' experience during the gameplay. Third, the paired-sample $t$-test was conducted to examine whether students' global perspectives had significant changes before and after the eTournament. Lastly, the correlation test was conducted between the global perspectives and students' experience during the gameplay. 
TABLE 1 | List of the SDGs.

\begin{tabular}{ll}
\hline SDG 1 & \multicolumn{1}{c}{ No Poverty } \\
\hline SDG 2 & Zero Hunger \\
\hline SDG 3 & Good Health and Well-being \\
\hline SDG 4 & Quality Education \\
\hline SDG 5 & Gender Equality \\
\hline SDG 6 & Clean Water and Sanitation \\
\hline SDG 7 & Affordable and Clean Energy \\
\hline SDG 8 & Decent Work and Economic Growth \\
\hline SDG 9 & Industry, Innovation, and Infrastructure \\
\hline SDG 10 & Reducing Inequality \\
\hline SDG 11 & Sustainable Cities and Communities \\
\hline SDG 12 & Responsible Consumption and Production \\
\hline SDG 13 & Climate Action \\
\hline SDG 14 & Life Below Water \\
\hline SDG 15 & Life On Land \\
\hline SDG 16 & Peace, Justice, and Strong Institutions \\
\hline SDG 17 & Partnerships for the Goals \\
\hline
\end{tabular}

\section{FINDINGS}

At the start of the two eTournaments, there were a total of 659 students from 46 home regions (see Figures 2, 3).

263 students finished all the stages and filled in both pre- and post-eTournament surveys, and 198 responses remain valid after the data filtering process mentioned above.

The internal reliability of the GPI is first tested. The result is shown in Table 2.

In this table, the subscale with Cronbach alpha over 0.7 is generally considered as acceptable (Nunnally, 1978) while 0.5 Cronbach alpha is also legitimate and acceptable with a short scale (Dall'Oglio et al., 2010). Since the Cronbach Alpha in subscales "intrapersonal identity" and "interpersonal social interaction" only consist of three and four items, respectively, the GPI in this study is thus considered as reliable for further analysis.

\section{Students' Gameplay Experience in PaGamO}

To examine the perceived effectiveness of the eTournaments, this study explored students' feedback on the eTournament through three variables: 1) level of enjoyment in the eTournament; 2) perceived improvement of SDG awareness after the game; and 3) frequency of question-attempt. The result is shown in Table 3 .

Overall, the students had a moderate level of enjoyment, which gave the mean score of 3.62 for the item "level of enjoyment in the eTournament." By contrast, they gave a higher mean score of 3.75 for the perceived improvement in their SDG awareness after the eTournament.

Regarding the students' autonomy of selecting SDG, the students in the first eTournament $(N=99)$ were assigned SDG randomly, while the students in the second eTournament $(N=118)$ were allowed to choose one. An independent samples $t$-Test was conducted to see if there is any significant difference.

Table 4 shows that students who could freely choose their SDG would have a higher level of enjoyment, increased from 3.09 to $4.06(\mathrm{t}(215)=-8.17, p<0.05)$. Similarly, they reported a higher score $(M=4.40)$ than students in the first eTournament $(M=2.98)$ in terms of "perceived improvement about their SDG awareness" ( $\mathrm{t}$ (215) = $-8.968, p<0.05))$.

To further examine the relation between students' level of enjoyment and the effectiveness of eTournament on different SDGs, this study further examined how the students' scores were distributed differently across 17 SDG in the second eTournament $(N=118)$.

Figures 4, 5 show that the awareness improvement in SDGs 16 $(M=5.00), 17(M=5.00)$ and $3(M=4.71)$ was most significant and that in SDGs $2(M=3.89), 13(M=4.00), 4(M=4.13)$ and 8 $(M=4.13)$ was least significant.

The students enjoyed most in SDG $16(M=4.75), 17(M=$ $4.40)$, and $1(M=4.33)$, but the least in $8(M=3.75), 9(M=3.75)$ and $11(M=3.75)$.

Before the study, it was assumed the students would enjoy more and perceive more improvement in SDG awareness if they chose to answer the questions related to particular SDG(s) more frequently. However, it is interesting to find that students in some SDGs play the game more frequently but feeling bad, and vice versa. For example, students in SDG 2 (Zero Hunger) reported a higher frequency of question-attempt $(M=207)$, but they give a lower score in the perceived improvement of SDG awareness $(M=3.89)$. By contrast, students with SDG 10 (Reduced inequalities) have a relatively high score in the improvement of SDG awareness, whereas there was very few question attempts $(M=94)$.

\section{Change of Global Perspectives After the eTournament}

To examine the change of students' global perspective, a pairedsample $t$-test on the pre- and post-survey results was conducted. The findings are shown in Table 5 and Figure 6.

As shown in Table 5, although the mean score of cognitive knowledge and interpersonal social interaction have been slightly increased after the eTournament, the changes of most sub-scales of global perspectives did not reach the significant level, except "intrapersonal affect." It is surprising to observe that "intrapersonal effect" was reduced from 4.35 to 4.16 after the eTournament, which indicates that the students show less acceptance of others' cultural perspectives and have a lower degree of emotional confidence to manage the intercultural conflict after the eTournament.

On the other hand, to examine the impact of SDG allocation on changes in students' global perspective, an independently sampled $t$-Test was conducted. The result is shown Table 6. 
TABLE 2 | Internal reliability of GPI.

\begin{tabular}{|c|c|c|c|}
\hline & Number of items & Cronbach alpha (pre-survey) & Cronbach alpha (post-survey) \\
\hline Cognitive knowing & 7 & 0.721 & 0.732 \\
\hline Cognitive knowledge & 5 & 0.775 & 0.817 \\
\hline Intrapersonal identity & 3 & 0.607 & 0.646 \\
\hline Intrapersonal affect & 3 & 0.779 & 0.726 \\
\hline Interpersonal Social interaction & 4 & 0.537 & 0.582 \\
\hline
\end{tabular}

Comparing with students of the first eTournament $(M=0.105)$, the mean difference before and after the eTournament has a significant decrease $(M=-0.188)$ in terms of "cognitive knowledge" (t $(208)=3.26, p<0.05)$. By contrast, a significant increase in "interpersonal social interaction" among students in the second eTournament (0.144) is observed, while the students' score dropped 0.283 in the first eTournament. Second, both groups of students reported a lower score in "intrapersonal affect" after the eTournament, while the decrease is significantly higher among students in the first eTournament $(-0.498)$ than those in the second eTournament $(-0.225)(\mathrm{t}(208)=-4.15, p<0.05)$.

\section{Students' Perception, Frequency of Question-Attempt, and Global Perspective}

To examine how students' learning experience affects their global perspective, this study conducted a Pearson's correlation test between the "change of students' global perspectives before and after the eTournament" and different variables about students' gameplay. The result is shown in Table 7.

This shows that the frequency of question-attempt of students has a significant correlation with the change of students' global perspectives in terms of "cognitive knowledge" $(r=0.250, p<0.05)$, "intrapersonal identity" ( $r=0.167, p<0.05)$, "intrapersonal affect" $(r=0.265, p<0.05)$, and "interpersonal social interaction" $(r=0.211, p<0.05)$.

The level of enjoyment is also found to have a significant correlation with "cognitive knowledge" $(r=0.167, p<0.05)$ and "interpersonal social interaction" $(r=0.177, p<0.05)$. However, the "perceived improvement of SDG awareness" has no correlation with all dimensions of global perspective, except "interpersonal social interaction $(r=0.239, p<0.05)$.

The result shows that the students who played $\mathrm{PaGamO}$ more frequently could gain more understanding about various cultures and their impact on our global society. They would have a higher degree of engagement with peers from other cultures and develop a higher degree of cultural sensitivity in living in pluralistic settings.

\section{DISCUSSION}

The results of the analyses indicate that students' gameplay experience is correlated with the development of students' global perspectives, while the gamified platform also created a desirable context for developing the global perspective. In this
TABLE 3 | Level of enjoyment, perceived awareness, and frequency of question attempts.

\begin{tabular}{lcc}
\hline & Mean & SD \\
\hline Level of Enjoyment & 3.62 & 0.99 \\
\hline Perceived improvement of UN-SDG awareness & 3.75 & 1.36 \\
\hline Frequency of question attempts & 145.0 & 74.7
\end{tabular}

section, the robustness of these findings is further discussed in comparison with existing literature.

\section{Students' Gameplay Experience and Perception in Different SDGs}

The above findings revealed that the eTournament has basically met its primary objective: to improve students' awareness of different SDGs through the eTournament. However, its change is probably affected by the nature of SDGs.

In the past, Begler (1993) suggested that knowledge inherent in a global perspective could be divided into substantive (Knowledge inherent in a global perspective) and perceptual domains (an array of intellectual values, dispositions, and attitudes). Begler suggested that perceptual domains offered the "lens" through which the substantive domain is viewed, while this study further revealed that these natures would also affect the effectiveness of the gamified learning experience. In Figure 4, it is observed that there is less improvement of SDG awareness in the substantive domains (i.e., SDG 2 "Zero hunger" and 13 "Climate Action"). It is probably because the students already had a relatively high awareness about the substantive challenges (i.e., impact greenhouse effect or global warming) through the advertisement of different non-governmental organizations (NGOs) or even popular disaster movies in the cinema. Moreover, people from developing and developed countries may have different concerns and led to different learning outcomes. Murakami et al., 2017, for example, reported that people in developed countries would have a higher environmental awareness of human health, biodiversity, and primary production. On the other hand, the news of famine and hunger, compared with other SDGs, would attract more attention from all over the world (De-Waal, 2017; Banik and Chasukwa, 2019) and raised enough awareness among students before the eTournament. 
TABLE 4 | Independent-sampled $t$-test between students in first and second eTournament.

\begin{tabular}{|c|c|c|c|}
\hline & $\begin{array}{l}\text { Mean (first eTournament, } \\
\qquad N=99)\end{array}$ & $\begin{array}{l}\text { Mean (second eTournament, } \\
\qquad N=118)\end{array}$ & $p$-Value \\
\hline Level of Enjoyment & 3.09 & 4.06 & 0.00 \\
\hline Perceived improvement of SDG awareness & 2.98 & 4.40 & 0.00 \\
\hline Frequency of question-attempt & 114.4 & 170.7 & 0.00 \\
\hline
\end{tabular}

TABLE 5 | Paired-sampled t-test of Students' Global perspectives in Pre- and Post-survey.

\begin{tabular}{lccc}
\hline & Mean-difference & t-Value & $\boldsymbol{p}$-Value \\
\hline Cognitive knowing & -0.06 & 1.14 & 0.256 \\
\hline Cognitive knowledge & 0.03 & -0.98 & 0.329 \\
\hline Intrapersonal identity & 0.00 & 0.04 & 0.970 \\
\hline Intrapersonal affect & -0.19 & 4.22 & 0.000 \\
\hline Interpersonal social interaction & 0.04 & 0.73 & 0.456
\end{tabular}

By contrast, the SDGs about perceptual domains, such as SDG 17 "Partnerships for the Goals" and 16 "Peace, Justice and Strong Institutions," were more complicated to be understood by the general public in daily life. Compared with the environmental issues, which were often portrayed in different disaster and action movies (Keane, 2006), the universal value (i.e., peace or justice) and global partnership may be less attractive to audience or more difficult to form intriguing storylines. Therefore, the students in these SDGs may receive greater shocks and perceived improvement of awareness during the gameplay.

Second, the comparison between the first and second eTournament in Findings showed that the students with greater autonomy in selecting their own SDG questions would have a significantly higher level of enjoyment, greater improvement of SDG awareness, and more question-attempt during the gameplay. It indicates that the game designer should pay more attention to the user's autonomy in selecting questions as it could greatly improve the attractiveness of gameplay.

Third, another point of view on students' varying levels of understanding of these SDGs may be related to recent criticisms.
The SDGs were recognized as being too complicated, inconsistent, and containing buzzwords without detailed definitions, such as "resilience" and "sustainable" (Liverman, 2018). In addition, in some SDGs, such as SDG 8 "Decent Work and Economic Growth" and SDG 10 "Reducing Inequality," there are certain conflicts such as boosting economic growth for the bottom 40\%, without considering the impact on the SDGs relating to the environment and reducing inequality by redistributing the top 1\%'s income (Liverman, 2018). This could make it difficult for learners to master these topics.

\section{The Effectiveness of eTournament in Promoting Students' Global Perspective}

Regarding the effectiveness of the eTournament on promoting students' global perspective, the result is somewhat surprising. Following the eTournament, students' levels of intrapersonal effect were found to be significantly lower. It is probably because most university students have overestimated their selfopenness to other cultures before real multicultural collaboration.

During the eTournament, the students would realize the incompatible views or different working styles of other team members. They have to manage unexpected cultural conflicts or even experience cultural shock during the interaction. Through the discussion process, the student could gain better self-understanding and identify their weakness in intercultural interaction.

In the age of globalization, however, university students should take risks and face challenges before they engage with foreigners as early as possible. It is the only way to enable students to develop advanced social skills and to manage the intercultural conflict through real intercultural interaction experiences, although such intercultural experience may not always be comfortable.

TABLE 6 | Independent-sampled t-test of Students' Global perspectives between first and second eTournament.

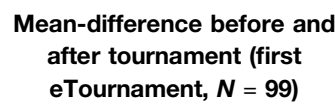

eTournament, $N=99$ )

\section{Mean-difference before and after tournament (second eTournament, $N=111$ )}

\begin{tabular}{lccc}
\hline Cognitive knowing & 0.105 & -0.188 & 3.26 \\
\hline Cognitive knowledge & -0.059 & 0.00 & 0.058 \\
\hline Intrapersonal identity & -0.074 & 0.024 & -1.56 \\
\hline Intrapersonal affect & -0.498 & -0.225 & -1.12 \\
\hline Interpersonal social interaction & -0.283 & 0.144 & -2.85
\end{tabular}



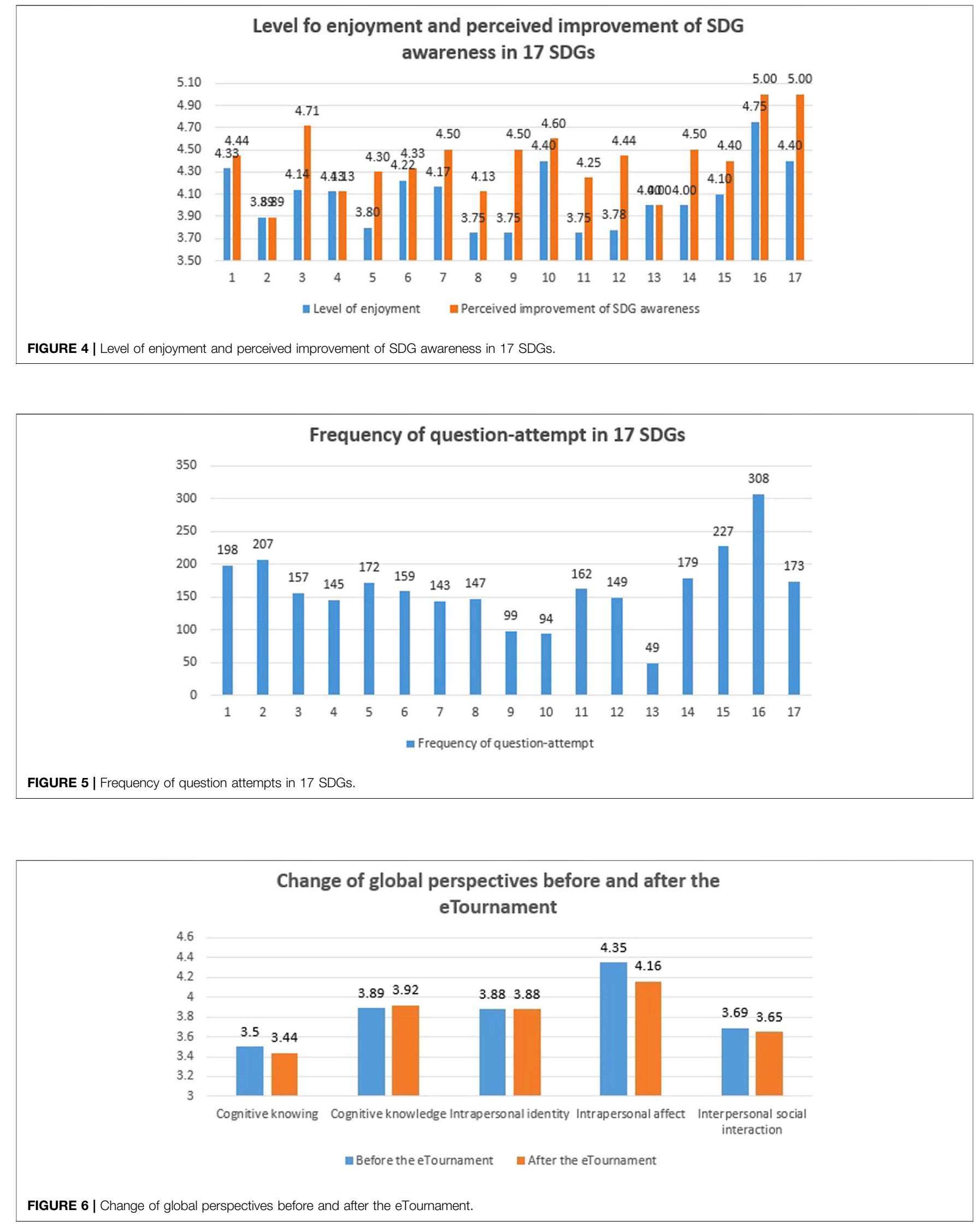
TABLE 7 | Correlation between students' global perspective, level of enjoyment, improved SDG awareness, and frequency of question attempts

\begin{tabular}{|c|c|c|c|}
\hline & Level of enjoyment & $\begin{array}{l}\text { Perceived improvement of } \\
\text { SDG awareness }\end{array}$ & Frequency of question-attempt \\
\hline Change of Cognitive knowing & 0.002 & -0.078 & 0.095 \\
\hline Change of Cognitive knowledge & $0.167^{\star}$ & 0.124 & $0.250^{*}$ \\
\hline Change of Intrapersonal identity & 0.067 & 0.079 & 0.167 \\
\hline Change of Intrapersonal affect & $0.199^{\star}$ & 0.111 & $0.265^{\star}$ \\
\hline Change of Interpersonal social interaction & $0.177^{\star}$ & 0.239 & $0.211^{*}$ \\
\hline
\end{tabular}

\section{Correlation Between the Students' Gameplay Experience and the Global Perspective}

The findings show that the eTournament could meet our learning objective, where students could gain more understanding and awareness of various cultures and their impact on our global society through more frequent gameplay. Moreover, the gameplay could also help students build up respect and acceptance to others with multicultural perspectives, as well as a higher level of emotional intelligence and cultural sensitivity for the unknown cultural conflict in the future.

In the eTournament, however, there are some limitations to the game design that we should be aware of. First, the eTournament could not help students to recognize the importance of cultural contexts or judge what the key knowledge or value in different SDGs is. It is also evident in our correlation test in Table 7, where no correlation is observed between the "change of cognitive knowledge" and all three variables about students' gameplay experience. It also indicates that the student participants could not develop the perspectivetaking ability that concerned other's unique identity after the eTournament. This is due to the fact that the tasks in this eTournament are rather simple and straightforward in the form of MCQ, and the students do not have to deal with complicated tasks during team collaboration.

Moreover, the results show that the perceived improvement of SDG awareness is not necessarily consistent with the students' global perspective. It indicates that the development of students' global perspective and students' awareness about SDG issues should be considered as two non-correlated variables. In other words, a multicultural team with stronger global perspectives would not guarantee a higher level of awareness about SDG. It is quite different from the traditional view that students with stronger global perspectives could have better quality collaboration with intercultural peers, and leads to more successful outcomes (Leinonen et al., 2005). It supports the suggestion of Leinonen and her colleagues that there is a need to investigate students' awareness of collaboration in more detail, including 1) awareness of the collaboration possibility, 2) awareness of the collaboration purpose, and 3) awareness of the collaboration process. It calls for the need to further explore the students' subjective perception and interpretation in the future, which may explain the missing link between multicultural collaboration and the expected learning outcomes, as shown in our study.

\section{CONCLUSION AND THE WAY FORWARD}

This study revealed that students' gameplay experience of the eTournament could contribute to their global perspective development. Regarding the impact of eTournament on 17 SDGs, the findings showed that students were satisfied with the level of enjoyment $(M=3.62)$ and perceived improvement of SDG awareness $(M=3.75)$ on average, while it was found to be particularly effective in improving perceptual dimensions of global perspectives in terms of issues about universal values (i.e., SDG 16 "Peace and Justice and Strong Institutions" and structured issues that involved multinational cooperation (e.g., SDG 17 "Partnerships for the Goals" or 3 "Good health and well-being." By contrast, the substantial dimensions (e.g., SDG 2 "Zero Hunger") or environmental issues SDG 13 "Climate Action"), were less likely to receive benefit from eTournament.

At the individual level, the findings showed that students become more aware of the difficulties of intercultural collaboration, such as integrating one's personal values and selfidentity into one's personhood. It is reflected in the decrease of "intrapersonal affect" after the eTournament, which indicates that students have re-estimated their level of respect for and acceptance of other cultural perspectives after the eTournament. It is believed that the students' experience in the eTournament could serve as a foundation for their future intercultural interactions towards a more comprehensive global perspective.

This study also examined which elements of gameplay contributed to most of the students' global perspectives development. The findings revealed that the level of students' cognitive knowledge and interpersonal social interaction was positively related to their level of enjoyment and frequency of question attempts. It helps future game designers and course instructors to realize the importance of motivating students' learning through gameplay experience and imposing more enjoyable elements to develop broader perspectives about the world.

From the instructors' point of view, this study gave insights into addressing the students' needs for different global issues. Before advocating the importance of global perspectives, it is better for universities to explore which global perspectives are 
better off for their online learning environment and which ones are not. For example, the findings of this study reveal that the students' awareness of SDG 3 "Good health and well-being", 16 "Peace, Justice and Strong Institutions," and 17 "Partnerships for the Goals" received the greatest improvement. The instructors could further explore more possibilities in their development in other game-based learning environments.

Second, as illustrated by the findings, despite most students reporting a satisfactory level of enjoyment and improvement in SDG awareness on average, the correlation is only observed between the change of global perspective, frequency of question-attempt, and level of enjoyment. This implies that game designers or instructors should show more concern about how to optimize the combination of gamified elements and the learning content when promoting students' global perspectives development.

The lack of identification of global perspectives among the participants through a qualitative approach is one of the limitations of this research. Despite the fact that the quantitative results showed students' learning gains and attitudes in this eTournament, there were no in-depth examinations to aid in the analysis of these results. As a result, future studies may concentrate on a different angle of inquiry, such as document reviews and content analysis similar to what McCabe, 1994 proposed, to identify the dimensions among these students' global perspectives. Another drawback of this study was that the research findings suggested that allowing students to selfselect SDG question sets in the eTournament may lead to increased motivation on learning the SDGs. Despite the results suggesting this rationale, there has been no further investigation into the exact point of students' shifts in attitude in a more valid manner.

In conclusion, this study reveals the possibility of developing students' global perspectives through a gamified e-learning platform. In the future, global education should begin to move from just an internationalized curriculum in the traditional classroom into the digital platform providing an opportunity to build up more in-depth global perspectives among students with multidisciplinary and multicultural backgrounds.

\section{REFERENCES}

Alexander, S. E., and Whitehouse, J. L. (2004). Challenges for Balancing Conservation and Development through Ecotourism: Insights and Implications from Two Belizean Case Studies. Sustainable Tourism, 76 129-142. doi:10.2495/ST040111

Baildon, M., Alviar-Martin, T., Bott, S., and Lam, M. (2018). “A Comparative Case Study of International Schools in Singapore and Hong Kong", in Competing Frameworks: Global and National in Citizenship Education. Editor A. Rapoport (Charlotte, NC: Information Age Publishing), 31-53.

Banik, D., and Chasukwa, M. (2019). The Politics of Hunger in an SDG Era: Food Policy in Malawi. Food Ethics 4 (2), 189-206. doi:10.1007/s41055-01900055-3

Barbosa, B., Santos, C., and Prado-Meza, C. M. (2020). There Is No One Way to Internationalization at home: Virtual Mobility and Student Engagement through Formal and Informal Approaches to Curricula. Revista Lusofona de Educacao 47, 85-98. doi:10.24140/issn.1645-7250.rle47.06

\section{DATA AVAILABILITY STATEMENT}

The datasets presented in this article are not readily available because they involve the privacy of individuals. Requests to access the datasets should be directed to FL, yickwah@hkbu.edu.hk.

\section{ETHICS STATEMENT}

The studies involving human participants were reviewed and approved by the Hong Kong Baptist University. The patients/ participants provided their written informed consent to participate in this study.

\section{AUTHOR CONTRIBUTIONS}

The first author conducted the literature review and wrote the manuscript with support from all other authors. The second author verified the data and supported the preparation of graphs and figures. The third author assisted with the revisions to the manuscript according to the reviewer's comments. All authors contributed to the design and implementation of the research.

\section{FUNDING}

The authors would like to thank the University Grants Committee of the Hong Kong Special Administrative Region and Hong Kong Baptist University for funding this project.

\section{ACKNOWLEDGMENTS}

The authors would like to thank the University Grants Committee of the Hong Kong Special Administrative Region and Hong Kong Baptist University for funding the CCGame Project (http://ccgame. hkbu.edu.hk/), from which this study originated.

Begler, E. (1993). Spinning Wheels and Straw: Balancing Content. Process, and Context in Global Teacher Education Programs. Theor. into Pract. 32 (1), 14-20. doi:10.1080/00405849309543567

Braskamp, L. A., Braskamp, D. C., and Engberg, M. E. (2014). Global Perspective Inventory (GPI): Its Purpose, Construction, Potential Uses, and Psychometric Characteristics. Chicago, IL: Global Perspective Institute.

Braskamp, L. A. (2009). Internationalization in Higher Education: Four Issues to Consider. J. Coll. Character 10(6). doi:10.2202/1940-1639.1688

Case, R. (1993). Key Elements of a Global Perspective. Soc. Edu. 57, 318-325.

Dall'oglio, A. M., Rossiello, B., and Coletti, M. F. (2010). Do healthy Preterm Children Need Neuropsychological Follow-Up? Preschool Outcomes Compared with Term Peers. Dev. Med. Child. Neurol. 52 (10), 955-961. doi:10.1111/j.1469-8749.2010.03730.x

de Wit, H., and Altbach, P. G. (2021). Internationalization in Higher Education: Global Trends and Recommendations for its Future. Pol. Rev. Higher Edu. 5, 28-46. doi:10.1080/23322969.2020.1820898

De-Waal, A. (2017). Mass Starvation: The History and Future of Famine. World Nutr. 9(1):70 doi:10.26596/wn.20189170-71

D. K. Deardorff (2009).in The SAGE Handbook of Intercultural Competence (Thousand Oaks, CA: SAGE). . 
Enonbun, O. (2010). Constructivism and Web 2.0 in the Emerging Learning Era: A Global Perspective. J. Strateg. Innovation Sustainability 6 (4), 16-25.

Evans, M., Ingram, A., MacDonald, A., and Weber, N. (2009). Mapping the Global Dimension of Citizenship Education in Canada: The Complex Interplay of Theory, Practice, and Context. Citizenship, Teach. Learn. 5 (2), 17-34.

Gibson, D., Knezek, G., Mergendoller, J., Garcia, P., Redmond, P., Spector, J. M., et al. (2011). "Performance Assessment of 21st century Teaching and Learning: Insights into the Future," in Proceedings of Society for Information Technology \& Teacher Education International Conference 2011. Editors M. Koehler and P. Mishra (Waynesville, NC, United States: Association for the Advancement of Computing in Education), 1839-1843.

Greenwood, D., Honey, M., and Clancy, A. (2016). Gaining a Global Perspective on Public Health through an International Student Nurse Collaboration. Jnep 6 (8), 123. doi:10.5430/jnep.v6n8p123

Guo, Y., Guo, S., Yochim, L., and Liu, X. (2021). Internationalization of Chinese Higher Education: Is it Westernization. J. Stud. Int. Edu. 1, 102831532199074. doi:10.1177/1028315321990745

Hassan, L. (2017). Governments Should Play Games. Simulation \& Gaming 48 (2), 249-267. doi:10.1177/1046878116683581

Hickel, J. (2019). The Contradiction of the Sustainable Development Goals: Growth versus Ecology on a Finite Planet. Sustain. Develop. 1, 1-12. doi:10.10022Fsd.194710.1002/sd.1947

Hong Kong Baptist University (2020). UN SDG International eTournament 2020. Retrieved from: http://ccgame.hkbu.edu.hk/sdg-etournament2020/

Hua, P. (2008). "Comparison between the People's Republic of China and the United States in the Field of Library and Information Science," in International and Comparative Studies in Information and Library Science: A Focus on the United States and Asian Countries. Editors Y. Q. Liu and X. Cheng (Lanham, MD: Scarecrow Press).

Hudson, T. D., and Tomás Morgan, R. (2019). Examining Relationships between Education Abroad Program Design and College Students' Global Learning. Frontiers 31 (2), 1-31. doi:10.36366/frontiers.v31i2.452

Keane, S. (2006). Disaster Movies: The Cinema of Catastrophe. 2nd ed. London: Wallflower Press.

Kendall, L. (2008). "The Conduct of Qualitative Interview: Research Questions, Methodological Issues, and Researching Online," in Handbook of Research on New Literacies. Editors J. Coiro, M. Knobel, C. Lankshear, and D. Leu (New York: Lawrence Erlbaum Associates), 133-149.

Kirkwood, T. F. (2002). Teaching about Japan: Global Perspectives in Teacher Decision-Making, Context, and Practice. Theor. Res. Soc. Edu. 30 (1), 88-115. doi:10.1080/00933104.2002.10473180

Kniep, W. M. (1986). Defining a Global Education by its Content. Soc. Edu. 50 (10), 437-466. doi:10.1023/A:1022994613635

Knight, J. (2004). Internationalization Remodeled: Definition, Approaches, and Rationales. J. Stud. Int. Edu. 8, 5-31. doi:10.1177/1028315303260832

Knight, J. (1994). Internationalization: Elements and Checkpoints. Canadian Bureau for International Education, Ottawa, Ontario, Canada. (CBIE)/ Bureau canadien de l'éducation internationale (BCEI) 7, 1-15http:// files.eric.ed.gov/fulltext/ED549823.pdf

Koivisto, J., and Hamari, J. (2019). The Rise of Motivational Information Systems: A Review of Gamification Research. Int. J. Inf. Manag. 45, 191-210. doi:10.1016/j.ijinfomgt.2018.10.013

Kopnina, H. (2015). The Victims of Unsustainability: A challenge to Sustainable Development Goals. Int. J. Sustain. Develop. World Ecol. 23 (2), 113-121. doi:10.1080/13504509.2015.1111269

Landorf, H., and Doscher, S. (2015). Defining Global Learning at Florida International University.AACU Diversity and Democracy. Washington, D.C., United States, Retrieved from: https://www.aacu.org/ diversitydemocracy/2015/summer/landorf.

Lavrakas, P. J. (2008). Encyclopedia of Survey Research Methods. London: SAGE. .

Leask, B. (2015). "Internationalizing the Curriculum," in Internationalizing the Curriculum. Editor E. Jones Abingdon 1st ed. (England: Routledge). doi:10.4324/9781315716954

Leinonen, P., Järvelä, S., and Häkkinen, P. (2005). Conceptualizing the Awareness of Collaboration: A Qualitative Study of a Global Virtual Team. Comput. Supported Coop. Work 14 (4), 301-322. doi:10.1007/s10606-005-9002-z
Liverman, D. M. (2018). Geographic Perspectives on Development Goals. Dialogues Hum. Geogr. 8, 168-185. doi:10.1177/2043820618780787

Mak, M. T. F., Wang, M., and Chu, S. K. W. (2019). Effects of a Gamified Learning Platform on Elementary School Students' Flow Experiences in Leisure reading. Melbourne, Australia: Paper presented at the ASIS \& T 2019 Annual Meeting.

McCabe, L. T. (1994). The Development of a Global Perspective during Participation in Semester at Sea: A Comparative Global Education Program. Educ. Rev. 46, 275-286. doi:10.1080/0013191940460305

Medora, N., Roy, R. N., and Brown, T. L. (2020). Students' Global Mindedness and Cultural Sensitivity: Examining the Implications of a Semester at Sea Study Abroad Voyage. Int. J. Teach. Learn. Higher Edu. 32, 305-317.

Merryfield, M. M. (1998). Pedagogy for Global Perspectives in Education: Studies of Teachers' Thinking and Practice. Theor. Res. Soc. Edu. 26 (3), 342-379. doi:10.1080/00933104.1998.10505855

Murakami, K., Itsubo, N., Kuriyama, K., Yoshida, K., and Tokimatsu, K. (2017). Development of Weighting Factors for G20 Countries. Part 2: Estimation of Willingness to Pay and Annual Global Damage Cost. Int. J. Life Cycle Assess. 23, 2349-2364. doi:10.1007/s11367-017-1372-1

Ng, J., and Nyland, B. (2016). "Internationalisation of Higher Education and Global Learning," in Global Learning in the 21st Century. Editors T. Barkatsas and A. Bertram (Rotterdam: SensePublishers)), 231-250. doi:10.1007/978-94-6300-761-0_13

Nunnally, J. C. (1978). Psychometric Theory. 2nd ed. New York: McGraw-Hill.

Oppenheim, A. (1992). Questionnaire Design, Interviewing and Attitude Measurement. London: Pinter.

Riaz, M. S., Cuenen, A., Janssens, D., Brijs, K., and Wets, G. (2019). Evaluation of a Gamified E-Learning Platform to Improve Traffic Safety Among Elementary School Pupils in Belgium. Pers Ubiquit Comput. 23 (5-6), 931-941. doi:10.1007/ s00779-019-01221-4

Seeber, M., Meoli, M., and Cattaneo, M. (2020). How Do European Higher Education Institutions Internationalize? Stud. Higher Edu. 45, 145-162. doi:10.1080/03075079.2018.1541449

Shephard, K., Bourk, M., Mirosa, M., and Dulgar, P. (2016). What Global Perspective Does Our university foster in Our Students?. Environ. Edu. Res. 23 (3), 398-414. doi:10.1080/13504622.2015.1126806

Smith, B., and Yang, W. (2017). Learning Outcomes in an Interdisciplinary Study Abroad Program: Developing a Global Perspective. J. Fam. Consum Sci. 109 (1), 43-50. doi:10.14307/jfcs109.1.43

Taşç, G. (2021). The Impact of COVID-19 on Higher Education: Rethinking Internationalization behind the Iceberg. Int. J. Curriculum Instruction 13, 522-536.

United Nations (2015). Sustainable Development Goals. Retrieved from: https:// sustainabledevelopment.un.org/?menu=1300.

Wang, V. X. (2009). Handbook of Research on E-Learning Applications for Career and Technical Education: Technologies for Vocational Training. Hershey, PA: Information Science Reference.

Warner, C. (2016). "Second and Foreign Language Education," in Foreign Language Education in the Context of Institutional Globalization. Editors N. van DeusenScholl 3rd ed (Cham: May Springer). doi:10.1007/978-3-319-02323-6

Conflict of Interest: The authors declare that the research was conducted in the absence of any commercial or financial relationships that could be construed as a potential conflict of interest.

Publisher's Note: All claims expressed in this article are solely those of the authors and do not necessarily represent those of their affiliated organizations, or those of the publisher, the editors, and the reviewers. Any product that may be evaluated in this article, or claim that may be made by its manufacturer, is not guaranteed or endorsed by the publisher.

Copyright $\odot 2021$ Leung, Lau, Wan, Law, Kwong and Wong. This is an open-access article distributed under the terms of the Creative Commons Attribution License (CC $B Y$ ). The use, distribution or reproduction in other forums is permitted, provided the original author(s) and the copyright owner(s) are credited and that the original publication in this journal is cited, in accordance with accepted academic practice. No use, distribution or reproduction is permitted which does not comply with these terms. 